\title{
Analysis of Whole Gut Motility for the Evaluation of Diabetic Gastroenteropathy: Still the Road Untraveled?
}

\author{
Eun Jeong Gong \\ Department of Internal Medicine, Gangneung Asan Hospital, University of Ulsan College of Medicine, Gangneung, Gangwon-do, Korea
}

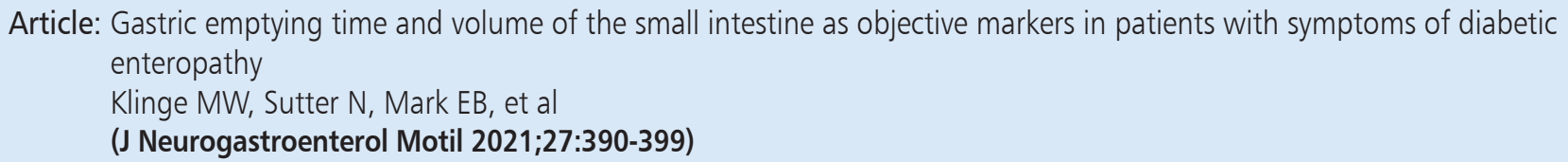

Diagnosis of diabetic gastroenteropathy is often challenging. Since diabetic gastroenteropathy can affect the entire gastrointestinal (GI) tract, the presenting symptoms associated with this condition, including nausea, vomiting, early satiety, fullness, and bloating are non-specific. ${ }^{1}$ The distinction between upper and lower GI involvement is not always clear, which further complicates the diagnostic approach in cases of diabetic gastroenteropathy.

Accordingly, a patient is diagnosed with diabetic gastroenteropathy through the exclusion of alternative diagnoses using laboratory tests, endoscopy, and imaging studies. Several functional tests are available for identification and quantification of bowel dysfunction; results of such tests can provide more detailed knowledge regarding the physiology and pathophysiology of GI neuromuscular function. ${ }^{2}$ However, there is a poor correlation between symptoms and objective findings of diabetic gastroenteropathy with respect to the optimization of diagnosis and treatment. ${ }^{1,3}$ Furthermore, the usefulness of measurement data in directing for the prediction of responses to therapy remains controversial.

In this issue of the Journal of Neurogastroenterology and Motility, a study investigating bowel transit abnormalities and the volume of small intestine in patients with type 1 diabetes mellitus (DM) has been published. ${ }^{4}$ GI motility was evaluated using the 3-dimensional (3D)-Transit system (Motilis Medica SA, Lausanne, Switzerland) in 20 patients with type $1 \mathrm{DM}$ who had symptoms that could be attributed to diabetic enteropathy and 20 matched healthy controls. The median gastric emptying time in the patients with DM was longer than that in the controls (3.3 hours and 2.4 hours, $P=0.002)$, whereas there was no significant difference between the median small intestinal transit time in the patients and that in the control groups (5.0 hours and 4.8 hours, $P=0.883$ ). Furthermore, the patients and healthy controls did not differ with respect to the frequency and amplitude of gastric contractions.

Additionally, gastric and small intestinal wall volumes were estimated through volumetric analysis of data obtained from CT scans. The volumes of the gastric wall and gastric contents were similar in the 2 groups, whereas total small intestinal volume and small intestinal wall volume were significantly larger in patients with DM than in controls. With respect to bowel content, compared to healthy controls, patients with DM appeared to have more fluid in the small intestine $(P=0.082)$; however, the difference was not statistically significant. The findings of the study suggest that the volume of small intestine may provide the most sensitive objective

Received: May 31, 2021 Revised: None Accepted: June 10, 2021

(a) This is an Open Access article distributed under the terms of the Creative Commons Attribution Non-Commercial License (http://creativecommons. org/licenses/by-nc/4.0) which permits unrestricted non-commercial use, distribution, and reproduction in any medium, provided the original work is properly cited.

*Correspondence: Eun Jeong Gong, MD, PhD

Department of Internal Medicine, Gangneung Asan Hospital, University of Ulsan College of Medicine, 38 Bangdong-gil, Gangneung, Gangwon-do 25440, Korea

Tel: +82-33-610-3106, Fax: +82-33-644-5495, E-mail: gong-eun@hanmail.net 
measure for diabetic enteropathy in patients with type $1 \mathrm{DM}$.

Gastric emptying scintigraphy using a standardized radiolabeled meal is the gold standard for the measurement of gastric emptying times. ${ }^{5}$ Scintigraphic assessment is also used to measure small bowel transit times. In a previous study, it was observed that among patients with gastroparesis, the number of patients with both delayed small bowel transit and delayed gastric emptying was greater than the number of patients with isolated delayed small bowel transit. ${ }^{6}$ This finding indicates that small bowel scintigraphy can be used in addition to gastric emptying tests to evaluate symptoms suspected of diabetic gastroenteropathy. ${ }^{6}$ However, limited normative data regarding small bowel transit times are available, and the interpretation of such data can be affected by abnormal gastric or colonic motility.

The wireless motility capsule is sensitive to $\mathrm{pH}$, temperature, and pressure changes in the GI tract, and it allows for an ambulatory and minimally invasive assessment of regional and whole gut transit times without radiation exposure. ${ }^{3}$ Although parameters evaluated using different modalities are not directly comparable to each other, the performance characteristics of the wireless motility capsule are considered reliable. ${ }^{7,8}$ A previous study in which gastric emptying scintigraphy and the wireless motility capsule were used found that extragastric or generalized transit delays occur in more than $40 \%$ of patients with gastroparesis, indicating that small bowel dysmotility may play an important role in gastroenteropathy. ${ }^{7}$

The 3D-Transit electromagnetic tracking system is another capsule-based technique that allows for an ambulatory assessment of gastric emptying, small intestinal transit time, and colonic transit times in a single investigation. ${ }^{9}$ A major advantage of the 3DTransit system is that it enables a detailed assessment of contraction patterns in a precise anatomical location. A previous study in which the 3D-Transit system was used showed that patients with type 1 DM had longer gastric emptying times, colonic transit times, and whole gut transit times than healthy controls. ${ }^{10}$

Imaging techniques such as MRI, positron emission tomography, and CT are also used for the morpho-functional assessment of the GI tract. ${ }^{11}$ Furthermore, attempts have been made to determine objective parameters, which can be useful surrogates for bowel dysfunction, through volumetric analyses. A recent study in which the small intestinal and colonic volumes in patients with type $1 \mathrm{DM}$ were evaluated using CT also showed that patients with DM were found to have larger small intestinal and colonic wall volumes that healthy controls. ${ }^{12}$ Similarly, in the present study, CT was utilized to estimate organ volumes, and the study showed that the small intestinal volume was larger in patients with suspected diabetic enteropathy than in controls. ${ }^{4}$ Despite the promising results of approaches involving volumetric analyses of the GI tract, analysis and interpretation of data extracted from imaging techniques are timeconsuming measures that cannot be used in current clinical practice. In addition, the clinical significance and underlying mechanisms of large small intestinal volumes, with respect to the development of symptoms, need to be further elucidated.

In conclusion, the measurement of gastric emptying times remains the standard for the assessment of patients suspected of having diabetic gastroenteropathy. Additional tests that involve the evaluation of whole gut motility may be useful complements to gastric emptying tests. However, given the lack of understanding about the pathophysiology of diabetic gastroenteropathy, it is uncertain whether information obtained through functional tests can influence management of patients with diabetic gastroenteropathy. The journey towards a better understanding of GI motility and dysmotility and the establishment of single method that is more desirable than other methods for a comprehensive assessment of diabetic gastroenteropathy is still underway.

\section{Financial support: None.}

\section{Conflicts of interest: None.}

\section{References}

1. DiBaise JK, Patel N, Noelting J, Dueck AC, Roarke M, Crowell MD. The relationship among gastroparetic symptoms, quality of life, and gastric emptying in patients referred for gastric emptying testing. Neurogastroenterol Motil 2016;28:234-242.

2. Parkman HP, Jones MP. Tests of gastric neuromuscular function. Gastroenterology 2009;136:1526-1543.

3. Arora Z, Parungao JM, Lopez R, Heinlein C, Santisi J, Birgisson S. Clinical utility of wireless motility capsule in patients with suspected multiregional gastrointestinal dysmotility. Dig Dis Sci 2015;60:1350-1357.

4. Klinge MW, Sutter N, Mark EB, et al. Gastric emptying time and volume of the small intestine as objective markers in patients with symptoms of diabetic enteropathy. J Neurogastroenterol Motil 2021;27:390-399.

5. Chedid V, Brandler J, Vijayvargiya P, Park SY, Szarka LA, Camilleri M. Characterization of upper gastrointestinal symptoms, gastric motor functions, and associations in patients with diabetes at a Referral Center. Am J Gastroenterol 2019;114:143-154.

6. Maurer AH, Yu D, Lu X, et al. Addition of small-bowel transit scintigraphy to gastric emptying for assessment of patients with upper gastrointestinal symptoms. Neurogastroenterol Motil 2021;33:e13987.

7. Hasler WL, May KP, Wilson LA, et al. Relating gastric scintigraphy and symptoms to motility capsule transit and pressure findings in suspected gastroparesis. Neurogastroenterol Motil Published Online First: 17 Jun 2018. doi: 10.1111/nmo.13196. 
8. Sangnes DA, Søfteland E, Bekkelund M, et al. Wireless motility capsule compared with scintigraphy in the assessment of diabetic gastroparesis. Neurogastroenterol Motil 2020;32:e13771.

9. Sutter N, Klinge MW, Mark EB, et al. Normative values for gastric motility assessed with the 3D-transit electromagnetic tracking system. Neurogastroenterol Motil 2020;32:e13829.

10. Klinge MW, Haase AM, Mark EB, et al. Colonic motility in patients with type 1 diabetes and gastrointestinal symptoms. Neurogastroenterol
Motil 2020;32:e13948.

11. Froehlich JM, Patak MA, von Weymarn C, Juli CF, Zollikofer CL, Wentz KU. Small bowel motility assessment with magnetic resonance imaging. J Magn Reson Imaging 2005;21:370-375.

12. Klinge MW, Borghammer P, Lund S, et al. Enteric cholinergic neuropathy in patients with diabetes: Non-invasive assessment with positron emission tomography. Neurogastroenterol Motil 2020;32:e13731. 